Research Article

\title{
Fuzzy Adaptive Control for Fractional Nonlinear Systems with External Disturbances and Unknown Control Directions
}

\author{
Yeguo Sun $\mathbb{D}^{1},{ }^{1}$ Yihong Liu, ${ }^{2}$ and $\mathrm{Ling}^{\mathrm{Li}}{ }^{3}$ \\ ${ }^{1}$ School of Finance and Mathematics, Huainan Normal University, 238 Dongshan West Road, Huainan 232038, China \\ ${ }^{2}$ School of Computer Science, Huainan Normal University, 238 Dongshan West Road, Huainan 232038, China \\ ${ }^{3}$ School of Economics and Management, Huainan Normal University, 238 Dongshan West Road, Huainan 232038, China
}

Correspondence should be addressed to Yeguo Sun; yeguosun@126.com

Received 12 November 2020; Revised 9 December 2020; Accepted 14 December 2020; Published 28 December 2020

Academic Editor: Ahmed Mostafa Khalil

Copyright (C) 2020 Yeguo Sun et al. This is an open access article distributed under the Creative Commons Attribution License, which permits unrestricted use, distribution, and reproduction in any medium, provided the original work is properly cited.

In this paper, the problem of fuzzy adaptive control of unknown nonlinear fractional-order systems with external disturbances and unknown control directions is studied. We exploit a decomposition of the control gain matrix into a symmetric positivedefinite matrix, a diagonal matrix with diagonal entries +1 or 1 , and a unity upper triangular matrix. Fuzzy logic systems are used for estimating the unknown nonlinear functions. Based on the fractional Lyapunov direct method and some proposed lemmas, a novel fuzzy adaptive controller is designed. The proposed method can guarantee that all the signals in the closed-loop systems remain bounded and the tracking errors converge to an arbitrary small region of the origin. In addition, for updating the parameters of the fuzzy system, fractional-order adaptations laws are proposed. Lastly, an illustrative example is given to demonstrate the effectiveness of the proposed results.

\section{Introduction}

Integer-order calculus has been generally accepted as an analytical mathematical tool for describing classical physics and related subject theories. The mathematical models of many problems can ultimately be attributed to the definite solution of integer-order differential equations, and integerorder calculus has a relatively complete theory both in theoretical analysis and numerical solution. But when people enter the study of complex systems and complex phenomena, the classical integer-order calculus equations will encounter some problems in the description of these systems. Based on the above reasons, people are eagerly looking forward to a usable mathematical tool and basic principles that can be used to model these complex systems. At this time, the fractional differential equation came into being for the reason that fractional calculus equations are very suitable for describing materials and processes with memory and hereditary properties. In systems such as diffusion, spectral analysis, and dielectrics, some mathematicians, physicists, and engineers have begun to apply fractional calculus to solve problems. In complex dynamic systems, the model established by using fractional calculus is often more accurate than the integer-order system model [1-5].

Fuzzy control expresses human experience and common sense in natural language by simulating human thinking, reasoning, and judgment and establishes an intelligent control model that is convenient for computer processing [6]. It is verified that fuzzy control has been successfully applied in various fields such as robot control, signal processing, medical care, power systems, and decision management [7]. On the other hand, the adaptive fuzzy control uses input and output data to adjust the process or controller parameters in real time by designing a suitable update law, which can be divided into direct and indirect fuzzy control [8]. In the direct fuzzy control method, the fuzzy system is directly approximated by the ideal control law, that is, the design goal is met by directly adjusting the control law parameters [9]. The indirect fuzzy control is based on the classic feedback linearization method [10]. Literature [11] studied the design of an adaptive fuzzy tracking controller with nonlinearity satisfying matching conditions. Literature 
[12] studied the problem of adaptive fuzzy output feedback control of a strict feedback system based on backstepping. Literature [13] uses integral type tangent function and studies the adaptive fuzzy output tracking control of a strict feedback system with multiple inputs and multiple outputs. For unknown nonlinear systems, adaptive fuzzy control shows great advantages, and its design method is simple and easy to understand, and it can be developed and designed using computer systems, so it has important research value in theoretical analysis and engineering applications. There are still many problems to be solved urgently, such as how to select a suitable fuzzy membership function, how to combine adaptive fuzzy control with other control methods to solve complex nonlinear system control, and how to deal with fractional-order controlled objects.

With the literature [14] proposing the second Lyapunov method of fractional-order systems, the control and stability analysis of fractional-order nonlinear systems has gradually become a research hot spot. For example, based on the theory of asymptotically autonomous systems and graph theory, the global Mittag-Leffler stability problem of the equilibrium point for a new fractional-order coupled system on a network without strong connectedness is investigated in [15]. By employing Lyapunov theory together with average dwell-time approach, the output tracking control for a class of fractional-order positive switched systems via an observer-based controller method that combines equivalentinput-disturbance approach and Smith predictor is studied in [16]. Based on the fractional Lyapunov stability theory, an adaptive fuzzy synchronization controller and a fractional parameter adaptive law are designed to realize the synchronization of an unknown chaotic system with asymmetric control gain in [17]. In [18], the authors have solved the issue of fuzzy adaptive controller design and system stability analysis of fractional-order uncertain nonlinear systems. In [19], the authors discussed the robust adaptive control for nonlinear uncertain fractional-order chaotic systems with external disturbances. In [20], the authors considered a class of nonlinear fractional-order systems with system uncertainty and external disturbances and designed a predetermined performance fractional-order fuzzy adaptive controller. In [21], the authors studied the fuzzy adaptive control problem of the fractional-order uncertain neural network when the neural network is subject to the dead zone and nonlinear input. In [22], the authors used adaptive control theory and Lyapunov direct method to discuss the synchronization control and parameter identification of a class of fractional time-delay neural networks. Based on the Lyapunov direct method and adaptive control theory, adaptive fuzzy control for a class of nonlinear fractionalorder systems is studied in [23].

On the basis of the aforementioned literature research, this paper mainly studies the tracking control of uncertain fractional-order chaotic systems with unknown control gain and external disturbance based on the adaptive fuzzy control method. Fuzzy logic systems are used to estimate unknown nonlinear functions. In order to solve the problem of adaptive fuzzy system parameters in fractional-order systems, a fractional-order adaptive law is designed. The asymmetric, sequential principle nonzero control gain matrix is decomposed into a known matrix with known diagonal elements of +1 or 1 and an unknown upper triangular matrix, where the unknown positive-definite matrix is used to construct the Lyapunov function, and the known diagonal matrix is used to construct a synchronous controller. The designed fuzzy adaptive controller can ensure that all variables of the closed-loop system are bounded and the tracking error approaches 0 asymptotically.

The main work of this article needs to be emphasized as follows. (1) This paper successfully uses fuzzy adaptive control to achieve the tracking control of fractional-order chaotic systems with external disturbance and unknown control direction. (2) For the first time, the unknown control gain problem is considered in the fractional-order system. In this paper, we only need that all sequential principles of the control gain matrix are nonzero. Therefore, the positivedefinite control gain matrix can be regarded as a special case of this paper. (3) The design of the novel adaptive law of the parameters for the fractional-order fuzzy system is proposed. (4) The squared Lyapunov function is used in the stability analysis, and the stability of the system is strictly proved. (5) The model of the fractional-order system in this paper can be completely unknown. In addition, using the method of this paper, many integer-order control methods can be extended to the systems described by fractional derivatives.

The remainder of this paper is organized as follows. In Section 2, problem formulation and some preliminaries are presented. Section 3 introduces the fuzzy logic systems. In Section 4, an adaptive fuzzy controller is designed and the stability of the closed-loop system is discussed by using fractional Lyapunov stability criterion. Simulation studies are presented in Section 5. Finally, Section 6 gives the conclusion of this work and the future research directions.

\section{Some Preliminaries and Problem Formulation}

In fractional calculus, scholars have given many definitions from different angles. In the research of mathematical theory and engineering applications, the commonly used derivates are Grunwald-Letnikov-type, Riemann-Liouville-type, and Caputo-type. Nevertheless, Caputo-type derivatives are the most widely used. However, Caputo-type derivatives are more suitable for actual initial value problems; on the other hand, Caputo-type derivatives are more similar to integerorder derivatives. Therefore, our paper focuses on the Caputo-type fractional-order system.

Definition 1 (Caputo-type fractional derivative). The Caputo-type fractional derivative of order $\alpha \in R^{+}$for the function $f(t)$ is defined as follows:

$$
D^{\alpha} f(t)=\frac{1}{\Gamma(n-\alpha)} \int_{a}^{t} \frac{f^{(n)}(\tau)}{(t-\tau)^{\alpha-n+1}} \mathrm{~d} \tau, \quad t>0,
$$

where $\Gamma(\cdot)$ is the Gamma function and the order $\alpha$ satisfies $n-1 \leq \alpha<n$. 
Definition 2 (Mittag-Leffler function). We define two parameters of the Mittag-Leffler function as follows:

$$
E_{\alpha, \beta}(z)=\sum_{k=0}^{\infty} \frac{z^{k}}{\Gamma(\alpha k+\beta)},
$$

where $z$ denotes a complex number and the parameters $\alpha$ and $\beta$ are positive.

Consider the following fractional-order nonlinear n-dimensional system with unknown control gain and external disturbances:

$$
\begin{array}{r}
D^{\alpha} x_{1}(t)=f_{1}(x(t))+\sum_{j=1}^{n} g_{1 j} u_{j}(t)+d_{1}(t), \\
D^{\alpha} x_{2}(t)=f_{2}(x(t))+\sum_{j=1}^{n} g_{2 j} u_{j}(t)+d_{2}(t), \\
\ldots, \\
D^{\alpha} x_{n}(t)=f_{n}(x(t))+\sum_{j=1}^{n} g_{n j} u_{j}(t)+d_{n}(t),
\end{array}
$$

where $x(t)=\left[x_{1}(t), x_{2}(t), \ldots, x_{n}(t)\right]^{T} \in R^{n}$ is the measurable system state vector, $u(t)=\left[u_{1}(t), \ldots, u_{n}(t)\right]^{T} \in R^{n}$ is the system control input, $d_{i}(t), i=1, \ldots, n$ are external interferences, $f_{i}(x), i=1, \ldots, n$ are nonlinear unknown functions, and $g_{i j}, i, j=1, \ldots, n$ are unknown control gains.

Denote

$$
\begin{aligned}
f(x(t)) & =\left[f_{1}(x(t)), \ldots, f_{n}(x(t))\right]^{T}, \\
d(t) & =\left[d_{1}(t), \ldots, d_{n}(t)\right]^{T}, \\
G & =\left(\begin{array}{ccc}
g_{11} & \cdots & g_{1 n} \\
\vdots & \ddots & \vdots \\
g_{n 1} & \cdots & g_{n n}
\end{array}\right) .
\end{aligned}
$$

Then, system (3) can be described as the following compact form:

$$
D^{\alpha} x(t)=f(x(t))+G u(t)+d(t) .
$$

Our main purpose is to design a fuzzy adaptive controller $u(t)$ so that under the action of the controller, the system state $x(t)$ can track the given reference signal well. In addition, all the relevant signals in closed-loop systems keep bounded.

Denote the reference signal as follows:

$$
x_{d}(t)=\left[x_{d 1}(t), x_{d 2}(t), \ldots, x_{d n}(t)\right] .
$$

Then, the system tracking error is

$$
e(t)=x_{d}(t)-x(t)
$$

Thus, the error system can be written as

$$
D^{\alpha} e(t)=D^{\alpha} x_{d}(t)-f(x(t))-d(t)-G u(t) .
$$

Assumption 1. The leading principal minors of control gain matrix are nonzero and their signs are also known.
Remark 1. It should be noted that Assumption 1 is meaningful. In fact, the control gain matrix in some practical systems such as visual servo system and automotive thermal management systems is asymmetric.

Assumption 2. The external disturbance is bounded, i.e.,

$$
\left|d_{i}(t)\right| \leq d_{i}, \quad i=1, \ldots, n,
$$

where $d_{i}, i=1, \ldots, n$ is an unknown positive constant.

Remark 2. It is worth noting that the restriction on $d_{i}(t)$ in Assumption 2 is not restrictive because we only need to assume that the external disturbance $d_{i}(t)$ has an upper bound, and it is not necessary to know the exact value of the upper bound $d_{i}$ in the controller design process.

Lemma 1 (see [2]). Suppose that $x(t)$ is both continuous and derivable; then,

$$
\frac{1}{2} D^{\alpha} x^{T}(t) P x(t) \leq x^{T}(t) P D^{\alpha} x(t),
$$

where the matrix $P \in R^{n \times n}$ is positive-definite.

Lemma 2 (see [24]). Any real matrix $G \in R^{p \times p}$ with nonzero leading principal minors can be decomposed as follows:

$$
G=G_{1} D T,
$$

where $G_{1} \in R^{p \times p}$ is a symmetric positive-definite matrix, $D \in R^{p \times p}$ is a diagonal matrix with +1 or 1 on the diagonal and $D D=I_{p \times p}$, in which $I_{p \times p}$ is identity matrix, and $T \in R^{p \times p}$ is a unity upper triangular.

Remark 3. It is noted that the decomposition of the control gain matrix $G$ in (11) is very important. In fact, the matrix $G$ is decomposed into an unknown positive-definite matrix, a known matrix with a known diagonal element of +1 or 1 , and an unknown upper triangular matrix, where the unknown positive-definite matrix of is used to construct the Lyapunov function, and the known diagonal matrix is used to construct the adaptive controller. In addition, if $G$ has nonzero leading principal minors, three cases can arise: if $G$ is positive-definite, then $D=I$. If $G$ is negative-definite, then $D=-I$. In the case where $G$ is indefinite, the matrix $D$ has +1 and 1 on the diagonal.

Lemma 3. If the state variable $y(t)$ satisfies the following fractional-order differential inequality:

$$
D^{\alpha} y(t) \leq-a y(t)+b
$$

then there exists a constant $t_{0}>0$ such that

$$
\|y(t)\| \leq \frac{2 b}{a}
$$

for all $t \in\left(t_{0}, \infty\right)$, where $a$ and $b$ are two positive constants.

Proof. In light of (12), there must exist a positive function $m(t)$ such that 


$$
D^{\alpha} y(t)=-a y(t)+m(t)+b .
$$

Taking Laplace transform on (14) gives

$$
Y(s)=\frac{s^{\alpha-1}}{s^{\alpha}+a} y(0)+\frac{E(m(t)+b)}{s^{\alpha}+a},
$$

where $y(0)$ is the initial state of $y(t)$. Then, we obtain

$$
y(t)=y(0) E_{\alpha, 1}\left(-a t^{\alpha}\right)+\int_{0}^{t}(t-\tau)^{\alpha-1} E_{\alpha, \alpha}\left(-a(t-\tau)^{\alpha}\right)(m(\tau)+b) \mathrm{d} \tau,
$$

which gives

$$
\|y(t)\| \leq\|y(0)\| E_{\alpha, 1}\left(-a t^{\alpha}\right)+b \int_{0}^{t}(t-\tau)^{\alpha-1} E_{\alpha, \alpha}\left(-a(t-\tau)^{\alpha}\right) \mathrm{d} \tau .
$$

In view of

$$
\int_{0}^{t} \tau^{\beta-1} E_{\alpha, \beta}\left(-a \tau^{\alpha}\right) \mathrm{d} \tau=t^{\beta} E_{\alpha, \beta+1}\left(-a t^{\alpha}\right),
$$

we can derive that

$$
\|y(t)\| \leq\|y(0)\| E_{\alpha, 1}\left(-a t^{\alpha}\right)+b t^{\alpha} E_{\alpha, \alpha+1}\left(-a t^{\alpha}\right),
$$

which means that there exists a constant $t_{0}>0$ such that for all $t \in\left(t_{0}, \infty\right),(13)$ is satisfied.

\section{Description of the Fuzzy Logic System}

The basic configuration of a fuzzy logic system consists of a fuzzifier, some fuzzy IF-THEN rules, a fuzzy inference engine, and a defuzzifier. The fuzzy inference engine uses the fuzzy IF-THEN rules to perform a mapping from an input vector $x=\left[x_{1}, x_{2}, \ldots, x_{n}\right]^{T} \in R^{n}$ to an output $\zeta(x) \in R$. The $i$ th fuzzy rule is written as follows.

Rule $i$ : if $x_{1}$ is $F_{1}^{i}$ and $\cdots$ and $x_{n}$ is $F_{n}^{i}$, then $\zeta(x)$ is $\alpha_{i}$, where $F_{1}^{i}, F_{2}^{i}, \ldots$ and $F_{n}^{i}$ are fuzzy sets and $\alpha_{i}$ is the fuzzy singleton for the output in the $i$ th rule. By using the singleton fuzzifier, product inference, and the center of gravity defuzzification, the output of the fuzzy system can be expressed as follows:

$$
\zeta(x)=\sum_{j=1}^{N} \alpha_{j} \frac{\prod_{i=1}^{n} \mu_{F_{i}^{j}}\left(x_{i}\right)}{\sum_{j=1}^{N}\left[\prod_{i=1}^{n} \mu_{F_{i}^{j}}\left(x_{i}\right)\right]=\theta^{T} \psi(x)}
$$

where $\mu_{F^{j}}\left(x_{i}\right)$ is the degree of membership of $x_{i}$ to $F_{i}^{j}, N$ is the number of fuzzy rules, $\theta=\left[\alpha_{1}, \ldots, \alpha_{N}\right]^{T}$ is the adjustable parameter vector, and $\psi(x)=\left[p_{1}(x), p_{2}(x), \ldots, p_{N}(x)\right]^{T}$, where

$$
p_{j}(x)=\frac{\prod_{i=1}^{n} \mu_{F_{i}^{j}}\left(x_{i}\right)}{\sum_{j=1}^{N}\left[\prod_{i=1}^{n} \mu_{F_{i}^{j}}\left(x_{i}\right)\right]}
$$

is the fuzzy basis function.

\section{Fuzzy Adaptive Controller Design}

In this section, we will design an adaptive fuzzy controller, such that not only all the signals of the closed-loop system (8) are bounded, but also the tracking error tends to the origin asymptotically. Based on the matrix composition (11), the dynamics of $e(t)$ can be rewritten as follows:

$$
G_{1}^{-1} D^{\alpha} e(t)=G_{1}^{-1} D^{\alpha} x_{d}(t)-G_{1}^{-1} f(x(t))-G_{1}^{-1} d(t)-D T u(t) .
$$

Let $P=G^{-1}$, and

$$
\mu(z(t))=\mu(x(t), u(t))=P D^{\alpha} x_{d}(t)-P f(x(t))-[D T-D] u(t) .
$$

Thus, (22) can be described as follows:

$$
P D^{\alpha} e(t)=\mu(z(t))-P d(t)-D u(t) .
$$

Considering that the external disturbance $d(t)$ in the system, the control gain matrix $G$, and the nonlinear function $f(x(t))$ are all completely unknown, the nonlinear function $\mu(z(t))$ is also unknown. Therefore, it is impossible to design an ideal controller. Nevertheless, we can design a fuzzy adaptive controller. In other words, we will approximate the unknown nonlinear function $\mu(z(t))$ based on the fuzzy system (20) in the following form:

$$
\widehat{\mu}_{i}\left(\theta_{i}(t), z(t)\right)=\theta_{i}^{T}(t) \psi_{i}(z(t)), \quad i=1,2, \ldots, n .
$$

Define the ideal parameters $\theta_{i}^{*}$ for $\theta_{i}$ as follows:

$$
\theta_{i}^{*}=\arg \min _{\theta_{i}}\left[\sup \left|\mu_{i}(z(t))-\widehat{\mu}_{i}(z(t))\right|\right] .
$$

Furthermore, define the parameter estimation errors and the fuzzy approximation errors as follows:

$$
\begin{aligned}
\tilde{\theta}_{i} & =\theta_{i}-\theta_{i}^{*}, \\
\varepsilon_{i}(z) & =\mu_{i}(z(t))-\widehat{\mu}_{i}\left(\theta_{i}^{*}, z(t)\right),
\end{aligned}
$$

where $\widehat{\mu}_{i}\left(\theta_{i}^{*}, z(t)\right)=\theta_{i}^{*} \psi_{i}(z(t))$. The fuzzy approximation error is assumed to be bounded for all $x$. That is to say, $\left|\varepsilon_{i}(z)\right|<\varepsilon_{i}^{*}$, where $\varepsilon_{i}^{*}$ is unknown constant. Denote $\varepsilon(z)=\left[\varepsilon_{1}(z), \ldots, \varepsilon_{n}(z)\right]^{T}, \varepsilon^{*}=\left[\varepsilon_{1}^{*}, \ldots, \varepsilon_{n}^{*}\right]^{T}$. Then, we can get $|\varepsilon(z)| \leq \bar{\varepsilon}$, which yields

$$
\begin{aligned}
\widehat{\mu}\left(\theta_{i}(t), z(t)\right)-\mu(z(t))= & \widehat{\mu}\left(\theta_{i}(t), z(t)\right)-\widehat{\mu}\left(\theta^{*}, z(t)\right) \\
& +\widehat{\mu}\left(\theta^{*}, z(t)\right)-\mu(z(t)) \\
= & \widehat{\mu}\left(\theta_{i}(t), z(t)\right)-\widehat{\mu}\left(\theta^{*}, z(t)\right)-\varepsilon(z(t)) \\
= & \widetilde{\theta}^{T}(t) \psi(z(t))-\varepsilon(z(t)) .
\end{aligned}
$$

We design a novel fuzzy adaptive controller as follows:

$$
u(t)=D\left[\operatorname{Ke}(t)+\theta^{T}(t) \psi(z(t))+E \operatorname{sign}(e(t))+F \operatorname{sign}(e(t))\right],
$$


where $K=\operatorname{diag}\left[k_{1}, \ldots, k_{n}\right]$ is a positive-definite matrix to be designed, $E=\operatorname{diag}\left[\widehat{\varepsilon}_{1}^{*}(t), \ldots, \widehat{\varepsilon}_{n}^{*}(t)\right]$ with $\widehat{\varepsilon}_{i}^{*}(t)$ is the estimation of the unknown constant $\varepsilon_{i}^{*}$, and $F=\operatorname{diag}\left[\widehat{d}_{1}^{*}(t), \ldots, \widehat{d}_{n}^{*}(t)\right]$ with $\widehat{d}_{i}^{*}(t)$ is the estimation of the unknown constant $d_{i}^{*}=\lambda_{\max }(P) d_{i}$. Substituting the proposed fuzzy adaptive controller (29) into error system (24) yields

$$
\begin{aligned}
P D^{\alpha} e(t)= & \mu(x(t))-P d(t)-K e(t)-\theta^{T}(t) \psi(z(t)) \\
& -E \operatorname{sign}(e(t))-F \operatorname{sign}(e(t)) .
\end{aligned}
$$

Multiplying $e^{T}(t)$ to both sides of tracking error dynamics (30) and applying (28) gives

$$
\begin{aligned}
e^{T}(t) P D^{\alpha} e(t) & \leq-e^{T}(t) K e(t)-\sum_{i=1}^{n} e_{i}(t) \tilde{\theta}_{i}^{T}(t) \psi_{i}(z(t))+\sum_{i=1}^{n}\left|e_{i}(t)\right| \varepsilon_{i}^{*}-\sum_{i=1}^{n}\left|e_{i}(t)\right| \widehat{\varepsilon}_{i}^{*}(t)+\sum_{i=1}^{n}\left|e_{i}(t)\right| \varepsilon_{i}^{*}-\sum_{i=1}^{n}\left|e_{i}(t)\right| \widehat{\varepsilon}_{i}^{*}(t) \\
& =-e^{T}(t) K e(t)-\sum_{i=1}^{n} e_{i}(t) \widetilde{\theta}_{i}^{T}(t) \psi_{i}(z(t))-\sum_{i=1}^{n}\left|e_{i}(t)\right| \widetilde{\varepsilon}_{i}^{*}(t)-\sum_{i=1}^{n}\left|e_{i}(t)\right| \widetilde{d}_{i}^{*}(t) .
\end{aligned}
$$

The control parameters $\theta_{i}(t), \widehat{\varepsilon}_{i}^{*}(t)$, and $\widehat{d}_{i}^{*}(t)$ are updated by the fractional-order differential equations as follows, respectively.

$$
\begin{aligned}
D^{\alpha} \theta_{i}(t) & =\mu_{i} e_{i}(t) \psi_{i}(z(t))-\mu_{i} \eta_{i} \theta_{i}(t), \quad i=1,2, \ldots, n, \\
D^{\alpha} \widehat{\varepsilon}_{i}^{*}(t) & =\sigma_{i}\left|e_{i}(t)\right|-\sigma_{i} \xi_{i} \widehat{\varepsilon}_{i}^{*}(t), \quad i=1,2, \ldots, n, \\
D^{\alpha} \widehat{d}_{i}^{*}(t) & =\gamma_{i}\left|e_{i}(t)\right|-\gamma_{i} \zeta_{i} \widehat{d}_{i}^{*}(t), \quad i=1,2, \ldots, n,
\end{aligned}
$$

where $\mu_{i}, \eta_{i}, \sigma_{i}, \xi_{i}, \gamma_{i}$, and $\zeta_{i}$ are positive parameters to be designed.

Remark 4. Fractional-order adaptation laws are also proposed to update the design parameters in in many existing references. However, it should be stressed that in the above literature, the right side of the fractional-order adaptation laws contains only a positive term. For example, the first adaptation law in (32) is $D^{\alpha} \theta_{i}(t)=\mu_{i} e_{i}(t) \psi_{i}(z(t))$, which leads to that the estimation of these design parameters will be monotone increasing. Thus, the boundedness of the design parameters is very hard to be ensured. In this paper, we add a term $-\mu_{i} \eta_{i} \theta_{i}(t)$ in the fractional-order adaptation laws. It is easy to find that the boundedness of the parameters to be updated can be guaranteed by choosing proper design parameters.

Theorem 1. Under Assumptions 1 and 2, if the controller and fractional adaptive law are designed as (29) and (32), respectively, then all relevant signals in the closed-loop system will keep bounded. Moreover, if all control parameters are chosen appropriately, the system tracking error will at last be arbitrarily small.
Proof. The quadratic Lyapunov function is constructed as follows:

$$
\begin{aligned}
V(t)= & \frac{1}{2} e^{T}(t) P e(t)+\frac{1}{2} \sum_{i=1}^{n} \frac{1}{\mu_{i}} \widetilde{\theta}_{i}^{T}(t) \widetilde{\theta}_{i}(t) \\
& +\frac{1}{2} \sum_{i=1}^{n} \frac{1}{\sigma_{i}} \widetilde{\varepsilon}_{i}^{* T}(t) \widetilde{\varepsilon}_{i}^{*}(t)+\frac{1}{2} \sum_{i=1}^{n} \frac{1}{\gamma_{i}} \widetilde{d}_{i}^{* T}(t) \widetilde{d}_{i}^{*}(t) .
\end{aligned}
$$

In view of Lemma 1 , we have

$$
\begin{aligned}
D^{\alpha} V(t) \leq & e^{T}(t) P D^{\alpha} e(t)+\sum_{i=1}^{n} \frac{1}{\mu_{i}} \widetilde{\theta}_{i}^{T}(t) D^{\alpha} \widetilde{\theta}_{i}(t) \\
& +\sum_{i=1}^{n} \frac{1}{\sigma_{i}} \widetilde{\mathcal{i}}_{i}^{* T}(t) D^{\alpha} \widetilde{\mathcal{\varepsilon}}_{i}^{*}(t)+\sum_{i=1}^{n} \frac{1}{\sigma_{i}} \widetilde{d}_{i}^{* T}(t) D^{\alpha} \widetilde{d}_{i}^{*}(t) .
\end{aligned}
$$

Based on the fact that the constant's Caputo derivative is equal to 0 , we can obtain

$$
\begin{aligned}
D^{\alpha} \widetilde{\theta}_{i}(t) & =D^{\alpha} \theta_{i}(t), \\
D^{\alpha} \widetilde{\varepsilon}_{i}^{*}(t) & =D^{\alpha} \widehat{\varepsilon}_{i}^{*}(t), \\
D^{\alpha} \widetilde{d}_{i}^{*}(t) & =D^{\alpha} \widetilde{d}_{i}^{*}(t) .
\end{aligned}
$$

Taking the fractional derivative of (33), we can obtain

$$
\begin{aligned}
D^{\alpha} V(t) \leq & e^{T}(t) P D^{\alpha} e(t)+\sum_{i=1}^{n} \frac{1}{\mu_{i}} \widetilde{\theta}_{i}^{T}(t) D^{\alpha} \theta_{i}(t) \\
& +\sum_{i=1}^{n} \frac{1}{\sigma_{i}} \widetilde{\varepsilon}_{i}^{* T}(t) D^{\alpha} \widetilde{\varepsilon}_{i}^{*}(t)+\sum_{i=1}^{n} \frac{1}{\gamma_{i}} \widetilde{d}_{i}^{* T}(t) D^{\alpha} \widehat{d}_{i}^{*}(t) .
\end{aligned}
$$


Substituting equation (31) and equation (32) into (36) yields

$$
\begin{aligned}
D^{\alpha} V(t) \leq & -e^{T}(t) K e(t)-\sum_{i=1}^{n} \eta_{i} \widetilde{\theta}_{i}^{T}(t) \theta_{i}(t)-\sum_{i=1}^{n} \xi_{i} \widetilde{\varepsilon}_{i}^{* T}(t) \widehat{\varepsilon}_{i}^{*}(t)-\sum_{i=1}^{n} \zeta_{i} \widetilde{d}_{i}^{* T}(t) \widehat{d}_{i}^{*}(t) \\
= & -e^{T}(t) K e(t)-\sum_{i=1}^{n} \eta_{i} \widetilde{\theta}_{i}^{T}(t) \widetilde{\theta}_{i}(t)-\sum_{i=1}^{n} \eta_{i} \widetilde{\theta}_{i}^{T}(t) \theta_{i}^{*}-\sum_{i=1}^{n} \xi_{i} \widetilde{\varepsilon}_{i}^{* T}(t) \widetilde{\varepsilon}_{i}^{*}(t) \\
& -\sum_{i=1}^{n} \xi_{i} \widetilde{\varepsilon}_{i}^{* T}(t) \varepsilon_{i}^{*}-\sum_{i=1}^{n} \zeta_{i} \widetilde{d}_{i}^{* T}(t) \widetilde{d}_{i}^{*}(t)-\sum_{i=1}^{n} \zeta_{i} \widetilde{d}_{i}^{* T}(t) d_{i}^{*} .
\end{aligned}
$$

In light of it is not difficult to draw that

$$
\begin{aligned}
& -\sum_{i=1}^{n} \eta_{i} \widetilde{\theta}_{i}^{T}(t) \theta_{i}^{*} \leq \frac{1}{2} \sum_{i=1}^{n} \eta_{i} \widetilde{\theta}_{i}^{T}(t) \widetilde{\theta}_{i}(t)+\frac{1}{2} \sum_{i=1}^{n} \eta_{i} \theta_{i}^{* T} \theta_{i}^{*}, \\
& -\sum_{i=1}^{n} \xi_{i} \widetilde{\varepsilon}_{i}^{* T}(t) \varepsilon_{i}^{*} \leq \frac{1}{2} \sum_{i=1}^{n} \xi_{i} \widetilde{\varepsilon}_{i}^{* T}(t) \widetilde{\varepsilon}_{i}^{*}(t)+\frac{1}{2} \sum_{i=1}^{n} \xi_{i} \varepsilon_{i}^{* T} \varepsilon_{i}^{*}, \\
& -\sum_{i=1}^{n} \zeta_{i} \widetilde{d}_{i}^{* T}(t) d_{i}^{*} \leq \frac{1}{2} \sum_{i=1}^{n} \zeta_{i} \widetilde{d}_{i}^{* T}(t) \widetilde{d}_{i}^{*}(t)+\frac{1}{2} \sum_{i=1}^{n} \zeta_{i} d_{i}^{* T} d_{i}^{*},
\end{aligned}
$$

$$
\begin{aligned}
D^{\alpha} V(t) & \leq-e^{T}(t) K e(t)-\frac{1}{2} \sum_{i=1}^{n} \eta_{i} \widetilde{\theta}_{i}^{T}(t) \widetilde{\theta}_{i}(t)+\frac{1}{2} \sum_{i=1}^{n} \eta_{i} \theta_{i}^{* T} \theta_{i}^{*}-\frac{1}{2} \sum_{i=1}^{n} \xi_{i} \widetilde{\varepsilon}_{i}^{* T}(t) \widetilde{\varepsilon}_{i}^{*}(t)+\frac{1}{2} \sum_{i=1}^{n} \xi_{i} \varepsilon_{i}^{* T} \varepsilon_{i}^{*}-\frac{1}{2} \sum_{i=1}^{n} \zeta_{i} \widetilde{d}_{i}^{* T}(t) \widetilde{d}_{i}^{*}(t)+\frac{1}{2} \sum_{i=1}^{n} \zeta_{i} d_{i}^{* T} d_{i}^{*} \\
& \leq-e^{T}(t) K e(t)-\frac{\eta}{2} \sum_{i=1}^{n} \widetilde{\theta}_{i}^{T}(t) \widetilde{\theta}_{i}(t)+\frac{1}{2} \sum_{i=1}^{n} \eta_{i} \theta_{i}^{* T} \theta_{i}^{*}-\frac{\xi}{2} \sum_{i=1}^{n} \widetilde{\varepsilon}_{i}^{* T}(t) \widetilde{\varepsilon}_{i}^{*}(t)+\frac{1}{2} \sum_{i=1}^{n} \xi_{i} \varepsilon_{i}^{* T} \varepsilon_{i}^{*}-\frac{\zeta}{2} \sum_{i=1}^{n} \widetilde{d}_{i}^{* T}(t) \widetilde{d}_{i}^{*}(t)+\frac{1}{2} \sum_{i=1}^{n} \zeta_{i} d_{i}^{* T} d_{i}^{*},
\end{aligned}
$$

where which yields

$$
\begin{aligned}
& \eta=\min \left\{\eta_{1}, \eta_{2}, \ldots, \eta_{n}\right\}, \\
& \xi=\min \left\{\xi_{1}, \xi_{2}, \ldots, \xi_{n}\right\}, \\
& \zeta=\min \left\{\zeta_{1}, \zeta_{2}, \ldots, \zeta_{n}\right\},
\end{aligned}
$$

$$
\begin{aligned}
D^{\alpha} V(t) \leq & -\frac{2 k}{\lambda_{\max }(P)} \frac{1}{2} e^{T}(t) P e(t)-\frac{\eta \mu}{2} \sum_{i=1}^{n} \frac{1}{\mu_{i}} \widetilde{\theta}_{i}^{T}(t) \widetilde{\theta}_{i}(t)+\frac{1}{2} \sum_{i=1}^{n} \eta_{i} \theta_{i}^{* T} \theta_{i}^{*}-\frac{\xi \sigma}{2} \sum_{i=1}^{n} \frac{1}{\sigma_{i}} \widetilde{\varepsilon}_{i}^{* T}(t) \widetilde{\varepsilon}_{i}^{*}(t) \\
& +\frac{1}{2} \sum_{i=1}^{n} \xi_{i} \varepsilon_{i}^{* T} \varepsilon_{i}^{*}-\frac{\zeta \gamma}{2} \sum_{i=1}^{n} \frac{1}{\gamma} \widetilde{d}_{i}^{* T}(t) \widetilde{d}_{i}^{*}(t)+\frac{1}{2} \sum_{i=1}^{n} \zeta_{i} d_{i}^{* T} d_{i}^{*} \\
\leq & -k_{0} V(t)+\frac{1}{2} \sum_{i=1}^{n} \eta_{i} \theta_{i}^{* T} \theta_{i}^{*}+\frac{1}{2} \sum_{i=1}^{n} \xi_{i} \varepsilon_{i}^{* T} \varepsilon_{i}^{*}+\frac{1}{2} \sum_{i=1}^{n} \zeta_{i} d_{i}^{* T} d_{i}^{*},
\end{aligned}
$$


where

$$
\begin{aligned}
\mu & =\min \left\{\mu_{1}, \mu_{2}, \ldots, \mu_{n}\right\}, \\
\sigma & =\min \left\{\sigma_{1}, \sigma_{2}, \ldots, \sigma_{n}\right\}, \\
\gamma & =\min \left\{\gamma_{1}, \gamma_{2}, \ldots, \gamma_{n}\right\}, \\
k & =\min \left\{k_{1}, k_{2}, \ldots, k_{n}\right\}, \\
k_{0} & =\min \left\{\frac{2 k}{\lambda_{\max }(P)}, \eta \mu, \xi \sigma, \zeta \gamma\right\} .
\end{aligned}
$$
that

By using Lemma 3 , a positive constant $t_{0}$ must exist such

$$
\|V(t)\| \leq \frac{\sum_{i=1}^{n}\left(\eta_{i} \theta_{i}^{* T} \theta_{i}^{*}+\xi_{i} \varepsilon_{i}^{* T} \varepsilon_{i}^{*}+\zeta_{i} d_{i}^{* T} d_{i}^{*}\right)}{k_{0}}
$$

which means that

$$
\|e(t)\| \leq \sqrt{\frac{2 \sum_{i=1}^{n}\left(\eta_{i} \theta_{i}^{* T} \theta_{i}^{*}+\xi_{i} \varepsilon_{i}^{* T} \varepsilon_{i}^{*}+\zeta_{i} d_{i}^{* T} d_{i}^{*}\right)}{k_{0} \lambda_{\min }(P)}},
$$

which gives that the error $\|e(t)\|$ will be arbitrarily small in $\left(t_{0},+\infty\right)$, as long as the control parameters $k_{i}, \mu_{i}, \sigma_{i}$, and $\gamma_{i}$ are selected large enough. In addition, it is not difficult to find that all the relevant signals in the closed-loop control plant will keep bounded.

Remark 5. It is worth pointing out that there are mainly three challenges and difficulties in this paper. Firstly, fractional calculus is still in the exploratory stage, and its theoretical framework needs to be further expanded and improved, which brings many difficulties to the stability analysis and controller design for the considered fractionalorder nonlinear chaotic systems. Secondly, the state orbit of a chaotic system will never repeat and it is extremely sensitive to initial values, which leads to the consequence that it is difficult to design suitable controller to achieve good performance for the considered fractional-order nonlinear chaotic systems. Lastly, there are relatively few mature numerical algorithms for fractional calculus. Especially under the premise of ensuring the reliability and accuracy of calculation, it is difficult to improve calculation efficiency and solve the problem of excessive calculation and storage of fractional differential equations.

\section{Numerical Simulations}

In this section, an illustrative numerical example will be given to confirm the theoretical results and show the applicability and effectiveness of the proposed fuzzy adaptive control method. Consider fractional-order nonlinear systems as follows [18].

$$
\begin{aligned}
& D^{\alpha} x_{1}=\sin \left(x_{1}(t)\right)+0.8 \ln \left(x_{2}^{2}(t)+\cos ^{2}\left(x_{2}(t)\right)\right), \\
& D^{\alpha} x_{2}=\cos \left(x_{2}(t)\right)-0.5\left|x_{1}(t)\right| .
\end{aligned}
$$

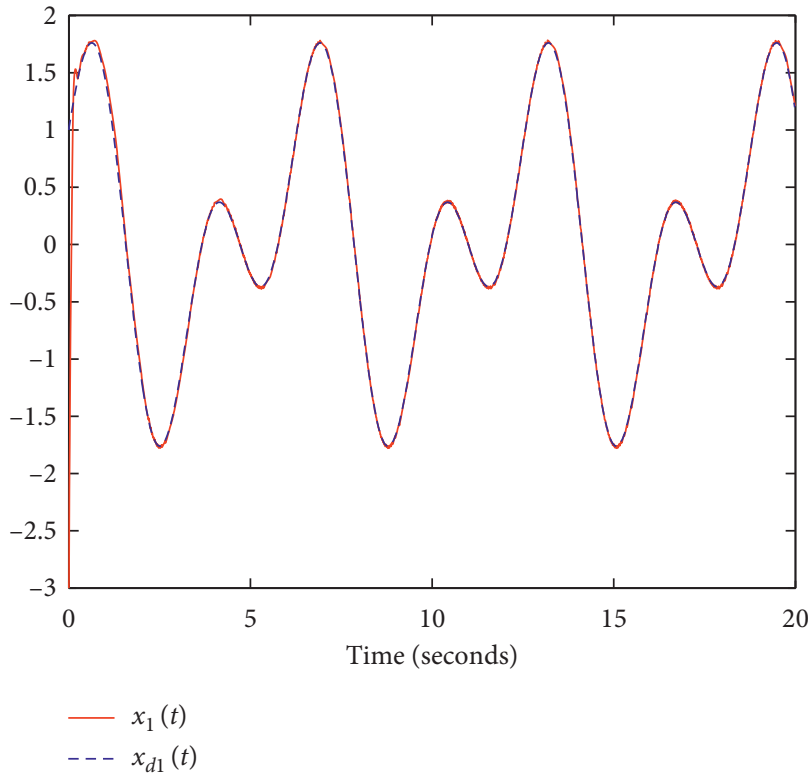

Figure 1: Trajectories of $x_{d 1}(t)$ and $x_{1}(t)$.

In the following simulation process, we assume that the external interferences $d_{i}(t), i=1,2$ and non-asymmetric control gain matrix $G$ are of the following form, respectively:

$$
\begin{aligned}
d_{1}(t) & =0.5 \sin (t), \\
d_{2}(t) & =0.4 \cos (t), \\
G & =\left(\begin{array}{cc}
1 & -0.2 \\
-0.1 & 0.65
\end{array}\right) .
\end{aligned}
$$

Obviously, the external disturbance is bounded and control gain matrix has nonzero leading principal minors, that is to say, Assumptions 1 and 2 are satisfied. Initial conditions of the system are selected as $x_{0}=[-3,-2]^{T}$. The referenced signal is set to be $x_{d}(t)=[\sin (2 t)+\cos (t)$, $\sin (t)+\cos (2 t)]^{T}$. Throughout the simulation, the model of the fractional-order nonlinear system (45) is fully unknown. The proposed control methods do not need the knowledge of the system. The states $x_{1}(t)$ and $x_{2}(t)$ are the inputs of the fuzzy systems, and we define 11 Gaussian membership functions uniformly distributed on $[-10,10]$ for each input. Thus, we know that there are $N=11 \times 11=121$ rules used in the simulation. The initial conditions for $\widehat{\varepsilon}_{i}^{*}(t)$ and $\widehat{d}_{i}^{*}(t)$ are chosen as $\widehat{\varepsilon}_{1}^{*}(0)=\widehat{\varepsilon}_{2}^{*}(0)=\widehat{d}_{1}^{*}(0)=\widehat{d}_{2}^{*}(0)=0.01$, and $\theta_{1}(0)$ and $\theta_{2}(0)$ are chosen randomly. The parameters of the controller are chosen as $k_{1}=k_{2}=1, \mu \quad \mu_{1}=\mu_{2}=\sigma_{1}=$ $\sigma_{2}=\gamma_{1}=\gamma_{2}=0.001, \eta_{1}=\eta_{2}=\xi_{1}=\xi_{2}=\zeta_{1}=\zeta_{2}=100$. The simulation results of the numerical example are shown in Figures 1-5.

Figures 1 and 2, respectively, show the tracking performance of the system states $x_{1}(t)$ and $x_{2}(t)$. Figure 3 depicts the response of the system tracking error, Figure 4 shows the control input trajectory of the system, and the boundedness of the fuzzy logic system parameters is included in Figure 5. From Figures 1 and 2, it is not difficult to find that under the action of the controller, the system states 


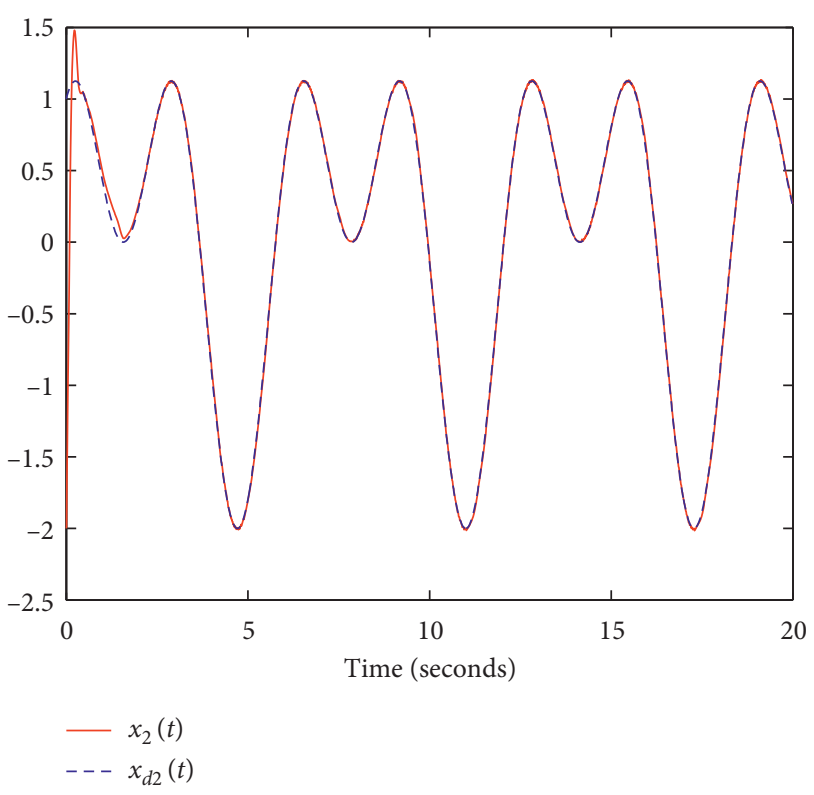

Figure 2: Trajectories of $x_{d 2}(t)$ and $x_{2}(t)$.

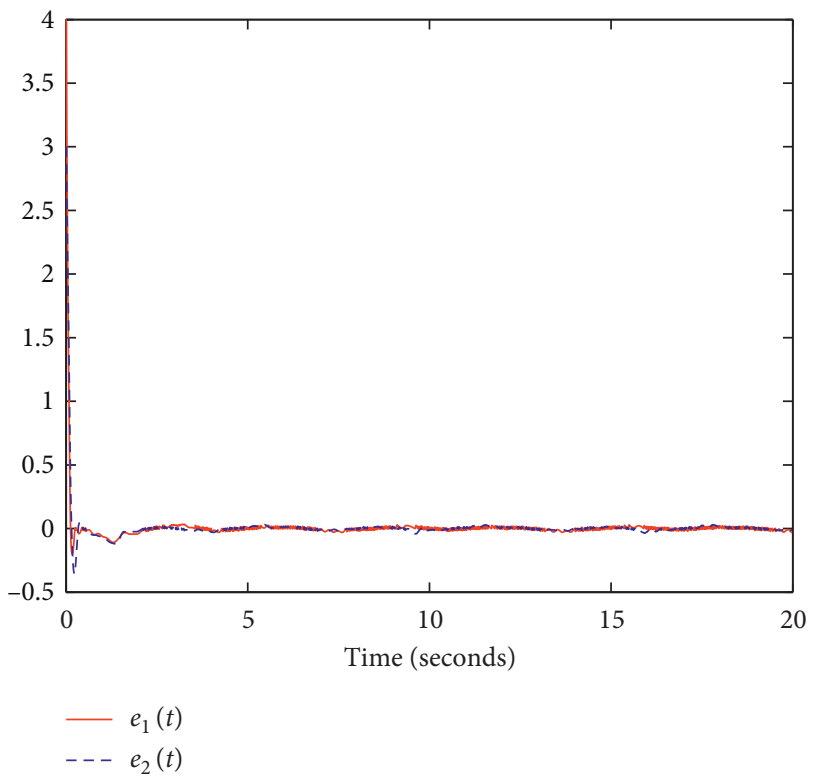

Figure 3: The tracking errors $e_{1}(t)$ and $e_{2}(t)$.

$x_{1}(t)$ and $x_{2}(t)$ can track the objective functions $x_{d 1}(t)$ and $x_{d 2}(t)$. From Figure 3 , we can see that the tracking error of the system quickly converges to zero. In summary, it can be seen from the simulation results that the control method we propose is effective. In addition, under the control of the controller designed in this paper, good control performances are achieved.

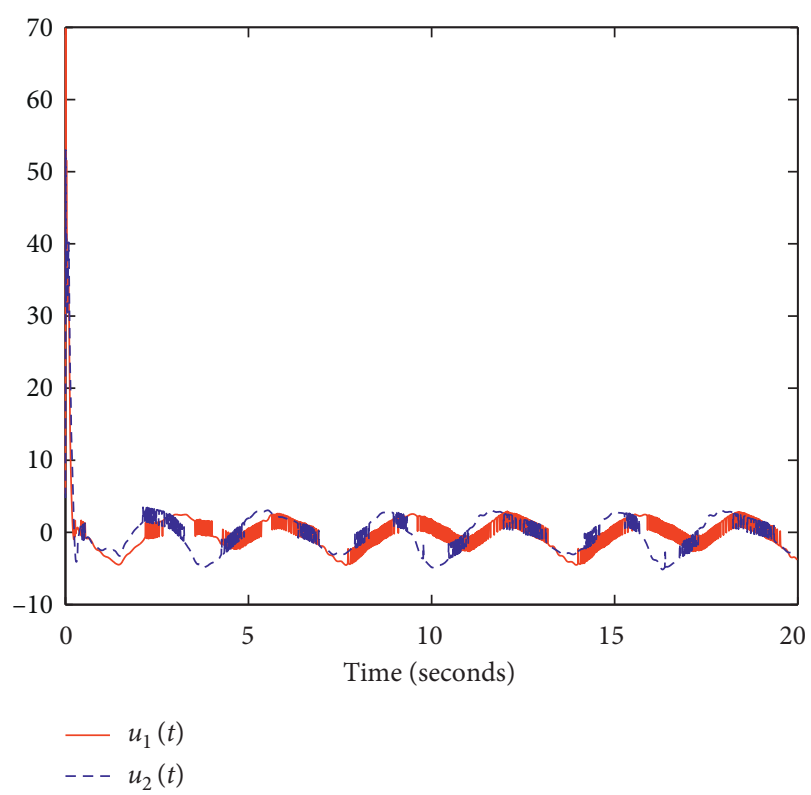

Figure 4: The control inputs $u_{1}(t)$ and $u_{2}(t)$.

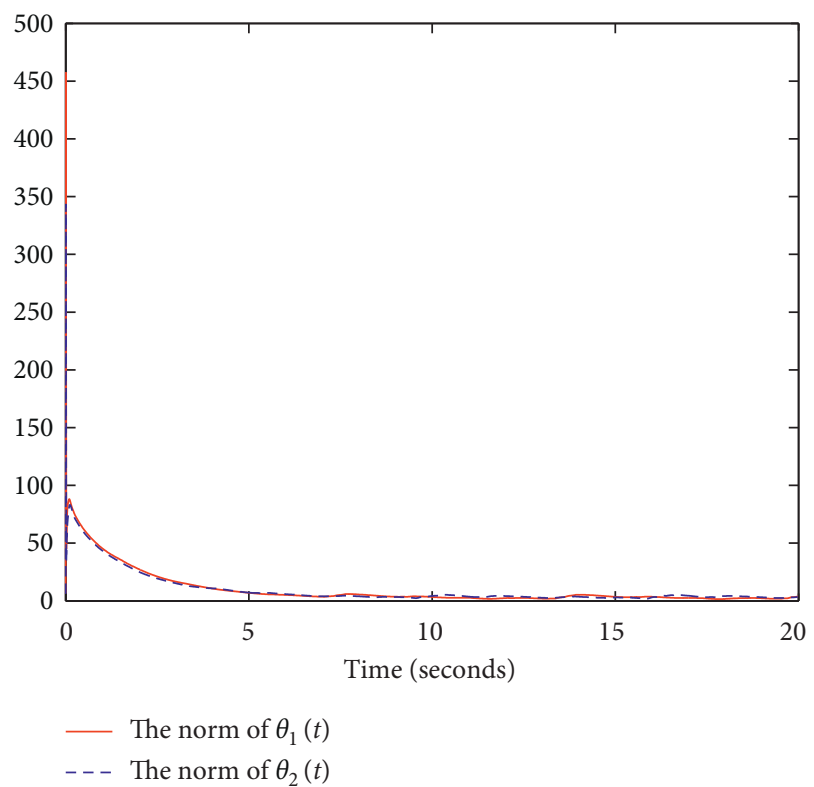

FIgURE 5: The norm of the fuzzy logic system parameters $\theta_{1}(t)$ and $\theta_{2}(t)$.

\section{Conclusions}

The problem of fuzzy adaptive control of unknown nonlinear fractional-order systems with unknown control direction and external disturbance is investigated in this paper. The control gain matrix is decomposed into a symmetric positive-definite matrix, a diagonal matrix with diagonal 
entries +1 or 1 , and a unity upper triangular matrix. Fuzzy logic systems are used for estimating the unknown nonlinear functions. Based on the Lyapunov direct method, a fuzzy adaptive controller is designed. The proposed method can guarantee that all the signals in the closed-loop systems remain bounded and the tracking errors converge to an arbitrary small region of the origin. A new fractional-order adaptation law is proposed. Lastly, we give an illustrative example to demonstrate the effectiveness of the proposed results. In practical systems, the state variables are usually unavailable, which makes it difficult to utilize them to stabilize the unstable systems $[25,26]$. In future work, based on the research results of this article, we will further study adaptive control of fuzzy systems with immeasurable premise variables via designing a suitable state observer.

\section{Data Availability}

The data used to support the findings of this study are included within the article.

\section{Conflicts of Interest}

The authors declare that they have no conflicts of interest.

\section{Acknowledgments}

This study was supported by the Anhui Provincial Natural Science Foundation (2008085MF200), Anhui Provincial University Natural Science Foundation (KJ2019ZD48), and Chinese National Natural Science Foundation (61403157).

\section{References}

[1] K. Diethelm, The Analysis of Fractional Differential Equations, Springer, Berlin, Germany, 2010.

[2] L. Zhang, Y. Yang, and F. Wang, "Synchronization analysis of fractional-order neural networks with time-varying delays via discontinuous neuron activations," Neurocomputing, vol. 275, pp. 40-49, 2018.

[3] T. Hu, X. Zhang, and S. Zhong, "Global asymptotic synchronization of nonidentical fractional-order neural networks," Neurocomputing, vol. 313, pp. 39-46, 2018.

[4] C. Huang, H. Liu, X. Shi et al., "Bifurcations in a fractionalorder neural network with multiple leakage delays," Neural Networks, vol. 131, pp. 115-126, 2020.

[5] Y. Sun and Y. Liu, "Fixed-time synchronization of delayed fractional-order memristor-based fuzzy cellular neural networks," IEEE Access, vol. 8, pp. 165951-165962, 2020.

[6] Y. Sun and L. Zhang, "Fixed-time adaptive fuzzy control for uncertain strict feedback switched systems," Information Sciences, vol. 546, pp. 742-752, 2021.

[7] C. Kahraman, M. Deveci, E. Boltürk, and S. Türk, "Fuzzy controlled humanoid robots: a literature review," Robotics and Autonomous Systems, vol. 134, Article ID 103643, 2020.

[8] J. WuY. Hu et al., "Indirect adaptive robust control of nonstrict feedback nonlinear systems by a fuzzy approximation strategy," ISA Transactions, 2020.

[9] N. Wang, J.-C. Sun, and Y.-C. Liu, "Direct adaptive self-structuring fuzzy control with interpretable fuzzy rules for a class of nonlinear uncertain systems," Neurocomputing, vol. 173, pp. 1640-1645, 2016.
[10] N. ShamlooA. Kalat et al., "Indirect adaptive fuzzy control of nonlinear descriptor systems," European Journal of Control, vol. 51, pp. 30-38, 2020.

[11] B. ChenC. Lee et al., " $H_{\infty}$ tracking design of uncertain nonlinear SISO systems: adaptive fuzzy approach," IEEE Transactions on Fuzzy Systems, vol. 4, no. 1, pp. 32-43, 1996.

[12] S. Tong, T. Wang, Y. Li, and B. Chen, "A combined backstepping and stochastic small-gain approach to robust adaptive fuzzy output feedback control," IEEE Transactions on Fuzzy Systems, vol. 21, no. 2, pp. 314-327, 2013.

[13] B. Chen, X. Liu, and S. Tong, "Adaptive fuzzy output tracking control of MIMO nonlinear uncertain systems," IEEE Transactions on Fuzzy Systems, vol. 15, no. 2, pp. 287-300, 2007.

[14] Y. Li, Y. Chen, and I. Podlubny, "Mittag-Leffler stability of fractional order nonlinear dynamic systems," Automatica, vol. 45, no. 8, pp. 1965-1969, 2009.

[15] X. Meng, Y. Kao, H. R. Karimi, and C. Gao, "Global Mittag-Leffler stability for fractional-order coupled systems on network without strong connectedness," Science China Information Sciences, vol. 63, no. 11, p. 132201, 2020.

[16] R. Sakthivel, S. Mohanapriya, C. K. Ahn, and H. R. Karimi, "Output tracking control for fractional-order positive switched systems with input time delay," IEEE Transactions on Circuits and Systems II: Express Briefs, vol. 66, no. 6, pp. 1013-1017, 2019.

[17] H. Liu, S.-G Li, Y.-G. Sun, and H.-X. Wang, "Adaptive fuzzy synchronization for uncertain fractional-order chaotic systems with unknown non-symmetrical control gain," Acta Physica Sinica, vol. 64, no. 7, Article ID 070503, 2015.

[18] H. Liu, S. Li, G. Li, and H. Wang, "Adaptive controller design for a class of uncertain fractional-order nonlinear systems: an adaptive fuzzy approach," International Journal of Fuzzy Systems, vol. 20, no. 2, pp. 366-379, 2018.

[19] L. Li and Y. Sun, "Adaptive fuzzy control for nonlinear fractionalorder uncertain systems with unknown uncertainties and external disturbance," Entropy, vol. 17, no. 8, pp. 5580-5592, 2015.

[20] H. Liu, S. Li, J. Cao, G. Li, A. Alsaedi, and F. E. Alsaadi, "Adaptive fuzzy prescribed performance controller design for a class of uncertain fractional-order nonlinear systems with external disturbances," Neurocomputing, vol. 219, pp. 422-430, 2017.

[21] H. Liu, S. Li, H. Wang, and Y. Sun, "Adaptive fuzzy control for a class of unknown fractional-order neural networks subject to input nonlinearities and dead-zones," Information Sciences, vol. 454-455, pp. 30-45, 2018.

[22] W. Ma, C. Li, Y. Wu, and Y. Wu, "Adaptive synchronization of fractional neural networks with unknown parameters and time delays," Entropy, vol. 16, no. 12, pp. 6286-6299, 2014.

[23] Y. Sun and L. Li, "Adaptive fuzzy control for a class of nonlinear fractional-order systems," in Proceedings of the 31st Chinese Control and Decision Conference, pp. 3477-3482, Nanchang, China, June 2019.

[24] A. Boulkroune, M. Tadjine, M. M'Saad, and M. Farza, "Fuzzy adaptive controller for MIMO nonlinear systems with known and unknown control direction," Fuzzy Sets and Systems, vol. 161, no. 6, pp. 797-820, 2010.

[25] M. Chadli and H. R. Karimi, "Robust observer design for unknown inputs Takagi-Sugeno models," IEEE Transactions on Fuzzy Systems, vol. 21, no. 1, pp. 158-164, 2013.

[26] B. Jiang, H. R. Karimi, Y. Kao, and C. Gao, "Adaptive control of nonlinear semi-markovian jump T-S fuzzy systems with immeasurable premise variables via sliding mode observer," IEEE Transactions on Cybernetics, vol. 50, no. 2, pp. 810-820, 2020. 